\title{
Téoros
}

Revue de recherche en tourisme

\section{La mise en scène du tourisme au Saguenay et au Lac-Saint-Jean (1842-1934)}

\section{Serge Gagnon}

Volume 19, numéro 1, printemps 2000

Pour une culture du tourisme au Saguenay-Lac-Saint-Jean

URI : https://id.erudit.org/iderudit/1071799ar

DOI : https://doi.org/10.7202/1071799ar

Aller au sommaire du numéro

Éditeur(s)

Université du Québec à Montréal

ISSN

0712-8657 (imprimé)

1923-2705 (numérique)

Découvrir la revue

Citer cet article

Gagnon, S. (2000). La mise en scène du tourisme au Saguenay et au Lac-Saint-Jean (1842-1934). Téoros, 19(1), 14-21.

https://doi.org/10.7202/1071799ar d'utilisation que vous pouvez consulter en ligne.

https://apropos.erudit.org/fr/usagers/politique-dutilisation/ 


\title{
toman
}

\section{LA MISE EN SCÈNE DU TOURISME AU Saguenay et Au LAC-SAInt-JeAn $(1842-1934)^{1}$}

\begin{abstract}
Il y a trente milles entre Jonquière et Saint-Bruno, et c'est à peu près au milieu de cette distance qu'intervient la ligne imaginaire séparant le comté du Lac-Saint-Jean du comté de Chicoutimi. De part et d'autre on tient à indiquer l'existence de cette démarcation, Chicoutimi ville et comté n'aimant guère être flanqués sur les bords du Lac, pas plus que les riverains de celui-ci n'aiment être confondus avec des voisins dont une semaine de voyage les séparait jadis (Bilodeau, 1924 : 62).
\end{abstract}

\section{Serge Gagnon}

Le touriste qui découvre l'entité administrative Saguenay-Lac-Saint-Jean pour la première fois est interpellé par la beauté des paysages, tant du Saguenay que du Lac-SaintJean. Mais, au-delà de leurs caractères spécifique, ces territoires, qui ont attiré, depuis plus de cent ans, des milliers d'adeptes de grands espaces, ont bénéficié de mises en valeur de nature différente. Comment certains acteurs socio-économiques (entreprises privées, gouvernement, etc.) peuvent-ils influencer ou favoriser le développement d'une région aux dépens d'autres territoires? Et quel est le rôle de ces mêmes intervenants dans le choix des destinations ? À partir de ce questionnement, le présent article propose de faire un portrait de la genèse et de la mise en scène du tourisme dans cette région.

L'initiative de «trajectoires » vers des lieux valorisés, tels que le Lac-Saint-Jean et le Saguenay et la «mise en réserve » de domaines touristiques par certains acteurs socio-économiques ont habituellement précédé le développement de moyens de transport adéquats (Boyer, 1980), d'où la question du rôle de ces infrastructures en regard de l'émergence des lieux de tourisme et de villégiature. Par exemple, dans Charlevoix, l'absence d'une liaison ferroviaire et routière pourrait avoir été davantage voulue que subie. L'isolement relatif de la sous-région de Murray Bay (Pointe-au-Pic) aurait participé d'une mise en réserve d'un territoire hautement valorisé, favorisant ainsi le « faire-vouloir » auprès d'une clientèle périodiquement oisive (Gagnon S, 1996 et 1998).

Il ne s'agit pas, évidemment, de nier le fait que les moyens de transport (maritime, ferroviaire, terrestre) aient favorisé le développement des sites touristiques, ni même que ce développement pouvait permettre de rentabiliser les infrastructures qui se multipliaient ; cette reconnaissance est primordiale pour comprendre l'activité économique. Au contraire, si les moyens de transport ont appuyé le développement du tourisme et de la villégiature, c'est que certaines conditions étaient déjà réunies. En d'autres termes, les domaines touristiques réservés ont créé l'appel d'une desserte infrastructurelle qui, par la suite, a permis de rentabiliser une occupation proportionnellement onéreuse.

Afin de reconstituer cette dynamique territoriale et de préciser la structuration spatiale à l'origine des domaines touristiques du Saguenay et du Lac-Saint-Jean, nous nous proposons ici d'étudier les différents itinéraires des touristes ainsi que les interrelations entre les acteurs en présence de 1842 à 1934. Cela permettra de mieux définir les enjeux géopolitiques d'appropriation et d'occupation des espaces concernés. Pour aborder les caractères spécifiques associés à ces enjeux, il est alors pertinent de dresser un parcours géohistorique du tourisme régional. Il s'agit de montrer les différentes phases de transformation et les processus «spatiodynamiques » qui ont structuré l'activité touristique. On pourra ainsi mieux comprendre les positions spatiales et les rôles géotouristiques respectifs du Saguenay et du Lac-Saint-Jean. Finalement, la reconstitution des processus de transformation et d'organisation spatiale du tourisme permettra d'établir des repères pour interpréter les différences « qualitatives » sousrégionales.

\section{LA PÉNÉTRATION DU “ROYAUME »(1842-1908)}

Mais aujourd'hui, la Compagnie du Richelieu, la plus importante des compagnies de navigation intérieure de la province de Québec, a établi un service régulier quotidien de magnifiques paquebots, entre Québec et les deux ports de Chicoutimi et de la Grande-Baie, qui fonctionne pendant sept mois de l'année. D'un autre côté, depuis 1888, une compagnie extrêmement entreprenante, qui a su passer à travers des obstacles insurmontables, a réussi à construire une ligne de chemin de fer qui s'étend de Québec à Roberval, du côté ouest, et jusqu'à Chicoutimi, du côté est (Québec, 1900 : 112).

La première manifestation du tourisme au Saguenay-Lac-Saint-Jean est aussi vieille que la région elle-même. Elle se serait produite avant la levée de l'interdit d'établissement permanent sur le territoire du Domaine du Roi (Traite de Tadoussac). Celui-ci était fermé à toute occupation depuis les débuts de la colonie (Gagnon S, 1996 : 100-103). Il est intéressant de constater qu'au moment où le Gouvernement colonial de Londres est sur le point de supprimer la « réserve foncière » de la Couronne et de permettre la pénétration du territoire par des trajectoires 
de colonisation, le North America, bateau à vapeur de la compagnie de navigation St. Lawrence Steamboat Company, aurait été le premier navire de passagers à se rendre à Chicoutimi en $1842^{2}$. À partir de cette période, c'est l'embryon des premiers fronts de villégiature qui s'esquisse (Dubé, 1986: 145).

Ces nouveaux bourgeois aménagent les grandes places d'eau du fleuve SaintLaurent et du Saguenay ${ }^{3}$. La navigation à vapeur commence à faire sentir ses effets sur le développement du tourisme vers cette région, qui se taille ainsi une place au sein des grands circuits touristiques nord-américains (Gagnon, 1992 : 108). Les départs vers le Saguenay sont toutefois exceptionnels, malgré le fait que des bateaux à vapeur sillonnent le fleuve depuis le début du XIX ${ }^{\mathrm{e}}$ siècle. En 1849, un service régulier entre Québec et le Saguenay « [...] débuta avec le Rowland Hill [...], lançant un service maritime qui allait durer plus d'un siècle » (Beattie, 1995 : 17). Ces excursions, pour découvrir les beautés du « fjord», atteignent une popularité sans pareil à compter de 1850 (Gagnon F, 1992 : 114). Le ballet des croisières s'organise autour de l'embouchure du Saguenay et débute ainsi le règne de la compagnie de navigation du Richelieu.

\section{TADOUSSAC : LA «TÊTE-DE-PONT »DE LA BOURGEOISIE CANADIENNE}

Pour James M. Lemoine, Tadoussac signifie un retour « au berceau de notre histoire ». En 1865, de grands bourgeois canadiens, les Price, les Forsyth, les Rhodes, les Gilmour, les Russell, les Campbell, les Brydges et les Radford, s'organisent en compagnie pour « exploiter les bains de mer » de Tadoussac (1872 : 348349 ) et y construisent le premier hôtel (Beattie, 1995 : 17). La construction de l'hôtel «érige » Tadoussac «en place d'eau de premier ordre » (Gagnon F, 1922 : 121). Tadoussac prend l'allure d'une véritable station balnéaire avec, en plus, une série de résidences secondaires autour de la baie. Lord et lady Dufferin en font même leur lieu de vacances dès 1873 (Beattie, 1995 : 17 ; Trépanier, 1988).

Cette station de villégiature, organisée par la compagnie de navigation du Richelieu, devient le point de départ des excursions sur le Saguenay. Au XIX ${ }^{\mathrm{e}}$ siècle, un tel

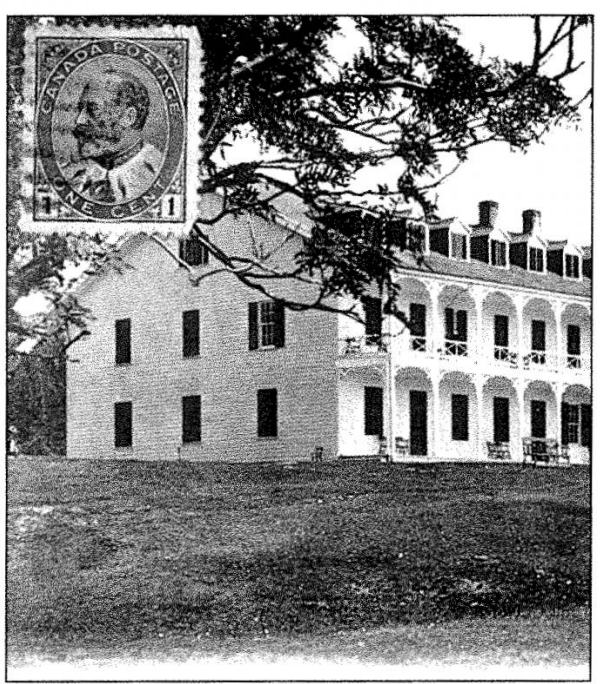

L'hôtel Tadoussac, commercialisé par plusieurs cartes postales dès le début de XX $X^{e}$ siècle, Archives nationales du Québec (Québec).

voyage est perçu comme étant très romantique. En 1872, James Macpherson Lemoine décrit ainsi cette aventure :

À l'homme d'étude, à l'homme d'affaires en villégiature, rien de plus rafraîchissant pour l'esprit et le corps, qu'un voyage sur le Clyde ou l'Union, en juillet ou en août, vers la source de la ténébreuse et profonde rivière qui arrose le vaste, le fertile territoire connu de nos pères sous le nom de Royaume du Saguenay $(1872: 351)$.

L'aristocrate décrit la croisière sur le Saguenay à une période particulièrement sensible, où l'on recherche l'émotion dans la réalisation de l'activité. C'est ainsi que Macpherson Lemoine suggère de faire le voyage par un temps couvert et orageux, pour saisir toute la magnificence des falaises qui surplombent le fjord.

[...] et ce beau désordre de la nature ne fera qu'ajouter à la majesté du spectacle qui vous attend [...] préparez-vous par le recueillement à subir les émotions que l'austère nature du Saguenay vous réserve [...] ce n'est plus un fleuve rangé, méthodique comme le vieux SaintLaurent, mais un ténébreux abîme, surplombé à certains endroits, comme aux Caps Trinité et Éternité, de promontoires [...] taillés à pic où des blocs de granit sont littéralement suspendus au-dessus du vapeur, à une hauteur de 1500 à 2000 pieds (1872: 352).

À la fin de son exposé, Il explique qu'il vient bien de communiquer une représentation imaginaire du Saguenay : «Tel est le Saguenay pour le touriste, il se présente tout autrement, sous son aspect historique, agricole et utilitaire » $(1872: 354)$. Cette affirmation permet de constater la force de l'évocation romantique qui masque l'objectivité utilitaire de l'espace.

En 1874, la grande bourgeoisie commence à intégrer les compagnies de navigation. Le rêve de contrôler la circulation maritime sur le Saint-Laurent pointe à l'horizon. La naissance de la Richelieu and Ontario Navigation Company (R\&O) permet d'offrir des croisières du Niagara jusqu'au Saguenay ${ }^{4}$.

Dix-huit navires battent son pavillon. On peu loger dans les hôtels de la compagnie, le luxueux Manoir Richelieu et l'Hôtel Tadoussac, davantage axé sur la famille. L'emprise de la $R \& O$ continua de s'affirmer sous la présidence du plus puissant homme d'affaires canadienfrançais de l'époque, Louis-Joseph Forget. Celui-ci accorda une place plus importante aux services aux passagers sur l'ensemble du réseau et notamment aux croisières sur le Saint-Laurent. En 1898, il fait agrandir le vieil Hôtel Tadoussac et, l'année suivante, il fait construire le premier Manoir Richelieu (Tittley, 1995 : 13).

La pénétration du « Royaume » par la voie des eaux est donc chose faite, l'acteur maritime personnifié par la $R \& O$ contrôlant 1'accès aux beautés du fjord via sa « tête-de-pont»: Tadoussac. À la même époque, un autre projet d'investissement territorial se profile, à savoir la « mise en réserve » du territoire du Lac-Saint-Jean pour fins de villégiature. D'autres acteurs font alors leur entrée en scène.

\section{L'AVENTURE FERROVIAIRE DU LAC-SAINT-JEAN}

En 1869, on conçoit le projet de relier la région du Lac-Saint-Jean par un chemin de fer. Cette liaison, à partir de Québec, en passant par le corridor de la rivière 
Batiscan, le lac Édouard et Chambord, soit un tracé de près de 260 kilomètres à travers le massif laurentien, n'atteindra Roberval qu'en 1888. Le point d'arrivée du train au Lac-Saint-Jean est très important ; il magnifie cette étendue d'eau et offre une vue panoramique remarquable. Un voyageur qui arrivait de son périple par le train via le lac Édouard décrivait ainsi son arrivée sur les hauteurs de Chambord :

At four-thirty of a placid afternoon on loosejointed train manoeuvred around the last curve above Chambord, and, as paradise opens out before a mystic's eyes, there unfolded before mine the blue, composure-blest, wide waters of St. John, and beyond, the dim, wild gateways to the space-begotten North (Longstreth, 1922 : 153).

Selon Arthur Buies, journaliste, pamphlétaire et vif défenseur du projet, la réalisation de la ligne a représenté « une odyssée, $[\ldots]$ presque douloureuse » (1886: 15). Sa construction progressive ouvre au fur et à mesure l'accès à un nouveau domaine aux chasseurs et aux pêcheurs, auxquels le gouvernement loue des territoires à des conditions très généreuses (Gagnon F, 1992).

L'instigateur du projet est un dénommé Horace-Jansen Beemer, financier américain de Pennsylvanie. Il correspond à l'image des grands entrepreneurs de l'époque. Promoteur de projets ferroviaires sur la rive nord du Saint-Laurent (actionnaire majoritaire du Montreal \& Occidental), celui-ci désire faire du lac Saint-Jean « un pays de vacances pour millionnaires » (Villeneuve, 1986:31) et « un des plus attrayants centres de voyage et de sport du Québec » (Gagnon R, 1978: 159). Sa compagnie, la société du Chemin de fer de Québec et du Lac-Saint-Jean, est incorporée en 1878.

Dès cette époque, les premiers signes de « mise en réserve » du lac Saint-Jean ne se font pas attendre. Avant même l'arrivée du train, le gouvernement accorde, en 1880, des droits de pêche exclusifs à la ouananiche sur le lac Saint-Jean, signe précurseur d'une prise de contrôle de ce territoire (Villeneuve, $1986: 31$ ). Beemer récupère ses droits dès son arrivée dans la région. En 1888, les lieux de pêche de la Grande Décharge « les trous » étaient loués par W.-A. Griffith et J.-G.-A. Creighton.
En 1889, appuyé par le conseil de village de Roberval, Beemer demande au gouvernement les droits de pêche dans toutes les rivières du bassin lacustre. Enfin, en 1890, Beemer en vient à régner sur toutes les eaux fréquentées par la ouananiche, « malgré certaines protestations locales »(Vien, $1954: 191)$.

\section{ROBERVAL : LA “ TÊTE-DE-PONT " DE BEEMER}

Tadoussac représentait, pour les acteurs de la navigation sur le Saint-Laurent, une porte d'entrée. À la confluence SaguenaySaint-Laurent, cette position permettait de garantir l'intégrité du domaine touristique, d'une part, en contrôlant la mobilité des touristes et, d'autre part, en assurant une surveillance de l'accès principal du Saguenay. De 1888 à 1908, Roberval a joué un rôle similaire pour la mise en valeur touristique du Lac-Saint-Jean. Étant la tête de ligne du chemin de fer et du transport sur le lac, Beemer choisit Roberval pour y construire, tout comme à Tadoussac, un hôtel de prestige « avec une vue imprenable sur le lac $\gg$ (Gaudreault, 1990: 48).

En 1876, Arthur Buies avait déjà prédit, dans ses récits de voyages, "qu'on verrait avant peu sur les rives du lac SaintJean, élégants hôtels, équipages princiers, chapeaux de soie luisant au soleil et chignons défiant les nues » (Lortie, 1993 : 54). Dans son histoire de Roberval, Russel Vien précise les intentions de Beemer : «En construisant le chemin de fer, il entrait dans ses plans d'ériger un vaste hôtel au terminus, sur les bords du lac » (1954: 189). Ouvert officiellement le 10 août 1888 , l'hôtel Roberval avait une capacité maximum de 150 personnes. En 1891, on l'agrandit à 257 chambres. Pour satisfaire pleinement sa clientèle de pêcheurs, Beemer aménage un second hôtel d'une trentaine de chambres, l'Island House, à l'entrée de la Grande Décharge.

La ligne de chemin de fer composait l'épine dorsale d'un réseau touristique bien orchestré (clubs de chasse et de pêche, hôtellerie au lac Saint-Joseph et au lac Édouard). Quant à son terminus, Roberval, il constituait le centre d'un circuit où Beemer proposait aux visiteurs des excursions au cœur de son domaine, le lac SaintJean, sur de luxueux vapeurs (Lebel, 1938). Les attraits proposés à cette époque seront mobilisés de nouveau au moment de la mi- se en place du « tour du lac Saint-Jean » en automobile, à partir des années vingt.

En plus du service quotidien de Roberval à la Grande Décharge, des excursions de pêche ou de chasse en tout lieu étaient toujours possibles [...] L'habitant chez lui, les Montagnais de Pointe-Bleue, le lac SaintJean, la chute de Val Jalbert : autant de simples attractions que Beemer exploitait (Vien, 1954 : 194).

On pratique également, à l'embouchure du lac, la descente des rapides de la Petite et de la Grande Décharge qui se jette dans la rivière Saguenay (Lortie, 1993 : 56).

En moins de dix ans, Roberval est passé d'un statut de petit village de colonisation à celui d'une ville avec tous les attributs d'un centre urbain.

On se servit de l'aqueduc en 1892, du téléphone en 1894, de l'électricité en 1897. L'année suivante était fondé le journal le Lac-Saint-Jean. Le Bureau d'enregistrement s'ouvrit en 1892, la Banque Nationale en 1895, soit trois ans seulement après la succursale de Chicoutimi (Gagnon R, 1978 : 159).

Cette remarque confirme que les positions touristiques sont porteuses « d'urbanité ».

Si l'on se place du point de vue de la hiérarchie urbaine, les modifications intervenues au XIX ${ }^{e}$ siècle dans les lieux de villégiature sont exceptionnelles. [...] Contrairement aux lois classiques de la diffusion de l'innovation, des lieux situés très bas dans la hiérarchie urbaine possédaient donc les attributs propres à la centralité des très grandes villes (Knafou, 1997 : 196).

Lors d'une conférence sur l'avancement des travaux du chemin de fer du Lac-SaintJean, en 1886, Buies décrivait la manœuvre de Beemer « sauveur du Lac-Saint-Jean » comme étant " un trait d'audace et une aventure admirablement calculée [...]» (Gagnon R, 1978 : 159). Cette aventure allait toutefois se frotter à un concurrent redoutable : la R\&O Navigation qui se positionnait du côté du Saguenay. 


\section{TADOUSSAC ET ROBERVAL : UNE BATAILLE RANGÉE ENTRE POSITIONS RIVALES}

Beemer avait attiré dans ses quartiers généraux la haute bourgeoisie américaine, mais aussi la noblesse européenne (Villeneuve, 1986 : 31). Profitant de la plus grande « mise en réserve » de pêche et de chasse au monde, cette clientèle sélecte était aussi sollicitée par les promoteurs du Saguenay, basés à Tadoussac. L'Américain Beemer établit ainsi une concurrence avec les stations «fashionables » du SaintLaurent.

Les amateurs de pêche du monde entier signent le registre: Paris, Bruxelles, Dublin, Londres, Moscou, Berlin, Tokyo. On concurrence sérieusement les Mille-îles et les stations balnéaires de l'estuaire du Saint-Laurent (Pépin, 1969 : 72).

L'Hôtel Roberval s'acquit immédiatement la renommée. [...] Le Progrès du Saguenay, en 1888, écrivait : Roberval fait pâlir La Malbaie, les deux cents îles de la Grande Décharge - naissance du Saguenaylaissent loin derrière elles les mille illes, plus ou moins bien comptées, du lac Ontario (Vien, 1954 : 190).

À la faveur de stratégies de promotion concurrentes, les bourgeoisies américaines (Roberval) et canadiennes (Tadoussac) se livrent donc une bataille rangée sur l'échi-

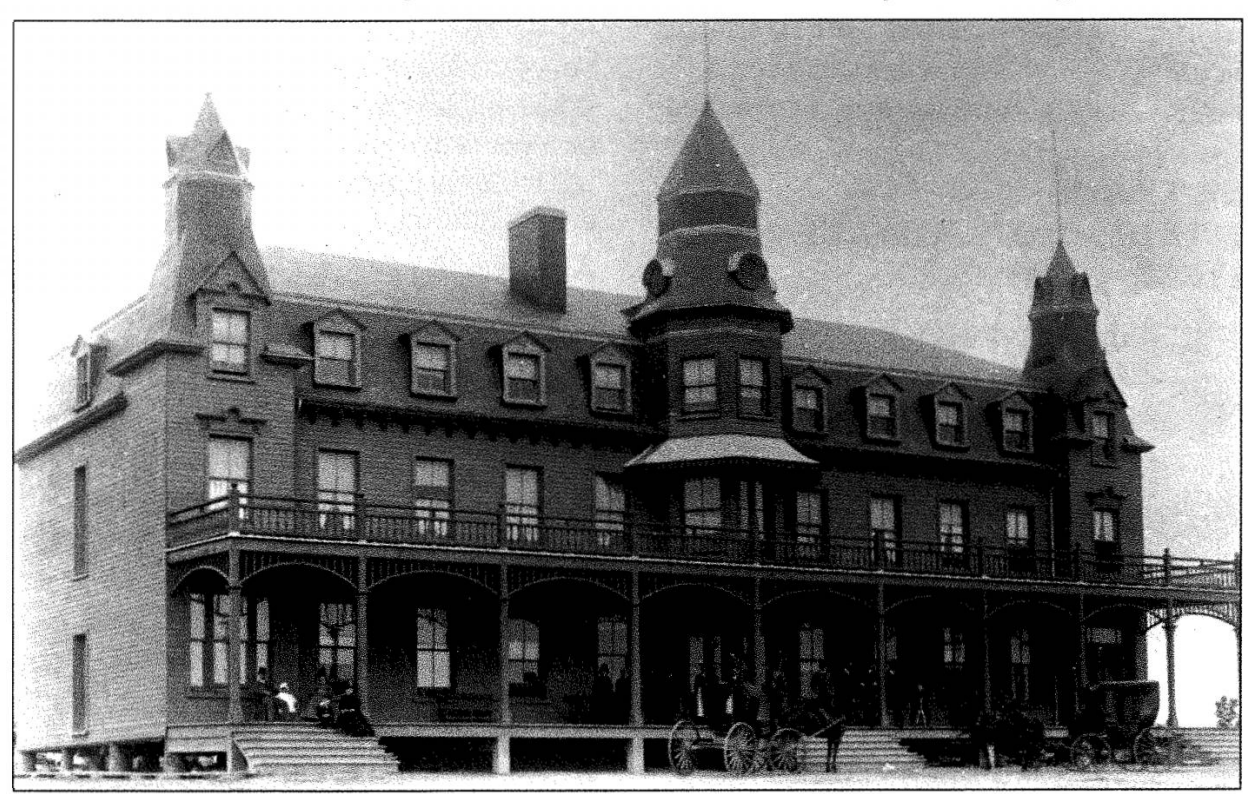

Hôtel Beemer de Roberval, Société d'archives Sagamie, Fonds de la Société historique du Sague-

nay, album Livernois.

quier géopolitique du tourisme international. Les acteurs mettent en branle une puissante machine publicitaire et tissent des alliances (voir tableau). Le Lac-SaintJean est à l'affiche dans les grands centres américains, où la compagnie de Chemin de fer de Beemer a des agences, tandis que le Saguenay trône fièrement dans les kiosques à bord des navires et dans les hôtels de la R\&O Navigation.

Les promoteurs font même appel à la plume d'écrivains réputés pour vanter les mérites de leur domaine respectif ou pour dénigrer leur adversaire. Voilà ce qu'Arthur Buies, allié irréductible de Beemer, déclarait lors d'une assemblée publique :

\section{[...] grâce à la construction du} chemin de fer du lac Saint-Jean, une immense région, absolument différente par son caractère sauvage de toutes celles où nous pénétrons en chemin de fer, va être offerte à la vil-

\begin{tabular}{|l|l|}
\hline \multicolumn{1}{|c|}{ Tadoussac } & \multicolumn{1}{c|}{ Roberval } \\
\hline - Hôtel prestigieux & - Hôtel prestigieux \\
- Accès exclusif (maritime) & - Accès exclusif (ferroviaire) \\
$\begin{array}{l}\text { - Émotion garantie (excursion sur le } \\
\text { fjord - caps Trinité et Éternité) }\end{array}$ & $\begin{array}{l}\text { - Émotion garantie (excursion sur le lac - pêche } \\
\text { à Grande Décharge) }\end{array}$ \\
\hline $\begin{array}{l}\text { Promoteur : bourgeoisie canadienne } \\
\text { Allié : gouvernement fédéral }\end{array}$ & $\begin{array}{l}\text { Promoteur : bourgeoisie américaine } \\
\text { (Gagnon R, 1978: 161) }\end{array}$ \\
\hline
\end{tabular}

légiature canadienne lasse de fréquenter éternellement les mêmes rivages du St-Laurent, et d'y attendre tous les jours l'heure bienfaisante de la marée pour prendre des bains. [...] Les amateurs de vraie campagne vont pouvoir s'en donner là à cour joie, avec abandon, avec volupté, affranchis qu'ils seront de toutes ces restreintes, de toutes ces petites tyrannies sociales auxquelles les assujettissent les stations d'eau fashionables, et, pour goûter ce parfait bonheur d'une villégiature sans mélange, complète, absolue, partout ailleurs irréalisable, ils n'auront qu'à faire deux ou trois heures de chemin de fer, le temps à peine de lire les journaux du jour (1886: 21-22).

En réaction à ce qu'avait écrit Arthur Buies, Damase Potvin, auteur du guide touristique The Saguenay Trip, lequel fut édité pendant plus de quarante années, relançait le débat :

Celui qui écrit ces lignes a toujours été un fervent admirateur d'Arthur Buies ; mais depuis qu'il a lu ce que Buies a écrit de Tadoussac, il a un peu moins d'estime pour la plume si alerte, si colorée de l'auteur du Saguenay. Arthur Buies, féru qu'il était de la Malbaie, a fait de Tadoussac une description aussi injuste que fantaisiste. Pour lui, Tadoussac n'a été qu'un roc velu, plein de trous et de bosses frissonnants aux vents du fleuve, qui abrite un reste de tribu indienne dans ses anfractuosités, quelques cottages dans ses replis et sur son dos et qui porte sur sa crête un hôtel fréquenté par des touristes qui n'ont pas le courage de se rendre jusqu'au Pôle Nord et qui confondent Tadoussac 


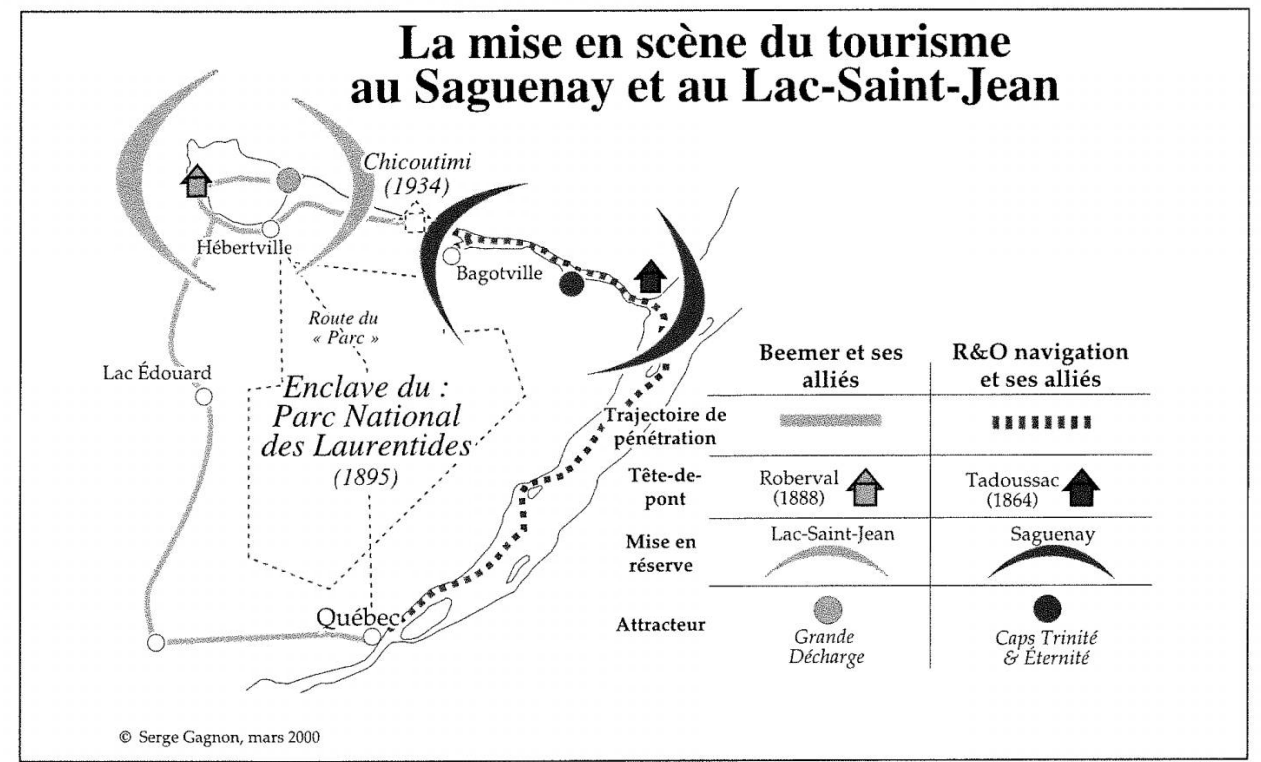

avec une station du Groenland. Ce sont là, tout simplement, calomnies atroces, et les centaines d'étrangers qui, chaque été, passent leurs vacances à Tadoussac auraient raison de demander à Buies de retirer ses paroles, s'il vivait encore (1920 : 8586).

À l'aube du XXe siècle, le « Royaume» était investi par les deux extrémités et les protagonistes s'étaient engagés dans la « mise en réserve » de leur domaine touristique respectif (voir figure). Cette dualité tire cependant à sa fin. Au cours de l'année 1908, l'hôtel Roberval brûle, réduisant en cendres tous les rêves de prospérité touristique du Lac-Saint-Jean.

En 1908, tous ces charmes existaient depuis vingt ans. Le sort impitoyable effaça tout d'un coup de Roberval le nom de Beemer : le Grand Hôtel Roberval passa au feu. Quand le couvent, le collège ou le quai brûle, on les reconstruit plus grands, mais quand l'Hôtel Roberval brûle, c'est fini (Vien, 1954 : 198).

Cette réflexion sur la subite disparition de «l'acteur » de la scène touristique soulève un questionnement qui déborde la simple destruction d'un édifice par les flammes. Pourquoi n'a-t-on pas reconstruit l'hôtel ? Celui-ci avait pourtant auréolé Roberval et le Lac-Saint-Jean d'une réputation inaltérable (Vien, 1954 : 194). Serait-il possible d'avancer que la $\mathrm{R} \& \mathrm{O}$ avait gagné la bataille du « Royaume » et que Beemer aurait « lancé la serviette »? L'incendie n'aurait alors été qu'un prétexte pour décamper. N'oublions pas que les compagnies de navigation, à cette époque, étaient en pleine période de gloire (Tittley, 1995 : 13).

Mentionnons aussi la compagnie Richelieu et Ontario, qui avec ses quinze steamers dont quelques-uns sont de véritables palais, dessert les ports du St-Laurent, pendant l'été, depuis Niagara jusqu'à Chicoutimi, tête de la navigation sur le Saguenay et qui donne un service au moins égal à celui des grandes compagnies américaines qui organisent des excursions pour les touristes riches (Québec, 1910 : 22).

Mais l'histoire n'est pas finie. L'État va maintenant entrer en scène avec son « joker » : le Parc national des Laurentides.

\section{LE TRACÉ D'UNE “ VOIE ROYALE " (1895-1934)}

Autrefois, ce nom de tour du Saguenay s'appliquait surtout au voyage en bateau comprenant la descente du Saint-Laurent jusqu'à Tadoussac et la remontée du Saguenay jusqu'à Chicoutimi, avec retour par le même trajet. Aujourd'hui, le véritable tour du Saguenay est celui que l'automobiliste peut faire en suivant l'itinéraire que nous avons tracé (Québec, $1934: 10)$.

Sous le couvert de l'idéologie de conservation, l'État entame une opération de « mise en réserve » d'un territoire et investit la zone centrale des terres de la Couronne.

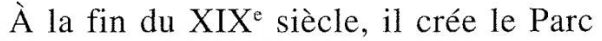
national des Laurentides, préfigurant ainsi le tracé d'une « voie royale » (la route Québec-Hébertville et ensuite le boulevard Talbot) qui le mènera au cœur du domaine réservé de la Canadian Steamship Lines à Chicoutimi. Au cours des années trente, l'État y établira sa «tête-de-pont » en y plaçant, en 1933, le siège d'une structure para-gouvernementale : le Syndicat d'initiative (ancêtre des actuelles Associations touristiques régionales).

\section{LE PARC NATIONAL DES LAURENTIDES : UNE OPÉRATION ÉTATIQUE DE « MISE EN RÉSERVE »}

Le Parc des Laurentides sera constitué en « réserve forestière » le 12 janvier 1895 , en même temps que le Parc de la Montagne-Tremblante au nord de Montréal. Des commentateurs de l'époque spécifiaient que le Parc des Laurentides représentait « probablement la plus grande réserve au monde à cette époque » (SaintAubin, 1987 : 19). Les articles numéros 1,2 et 4 de la Loi sanctionnant sa réalisation précisaient, respectivement, les limites de la zone qui sera publique, le statut de la réserve et les droits d'accès à ce territoire :

Article $n^{0} 1:$ : [...] toute la partie des terres vacantes de la couronne dans la province de Québec, situées dans les comtés de Montmorency, Québec et Charlevoix $[\ldots]$ le tout formant une superficie de 2,531 milles carrés plus ou moins, mis à part par ordre en conseil du six novembre mil huit cent quatre-vingt-quatorze, ne peut être vendu ou occupé [...] »;

$\operatorname{Article~}^{\circ} \mathbf{2}$ : «Ce territoire est mis à part comme réserve forestière, endroit de pêche et de chasse, parc public et lieu de délassement, sous le contrôle du commissaire des terres de la couronne, pour les citoyens de la province, sujet aux dispositions de cette loi et aux règlements qui seront faits en vertu d'icelle, et sera connu sous le mon de Parc national des Laurentides" ;

Article $^{\circ} 4$ : « Nul, sauf les personnes ayant bail, licence ou permis, ne peut s'établir ou se fixer sur, se servir de ou occuper aucune partie du parc, et aucun bail, licence ou permis, qui diminue ou puisse diminuer l'utilité du parc, ne peut être fait, accordé ou émis » (cité dans CIFQ, 1974). 
Par cet acte de propriété, l'État venait de rendre effective l'existence d'une « mise en réserve » dont il pouvait gérer les destinées à sa convenance. Paradoxalement, il avait fermé les accès terrestres tout juste avant la constitution de la réserve. Effectuons un retour dans le temps afin de comprendre cet enjeu.

\section{L'AVENTURE ÉPHÉMÈRE DE LA ROUTE DU "PARC " ET L'ENCLAVEMENT DES LAURENTIDES DE QUÉBEC}

En 1866, le parcours entre Métabetchouane et Québec est ouvert comme chemin d'hiver. Débute alors l'aventure de la route du «Parc ». Dès la fin des années 1860 , colons et touristes aventureux s'engagent dans cette traversée. Malgré la popularité de cette artère de communication, le gouvernement émet, en 1883, un avis à l'effet que le chemin de Métabetchouane ne sera plus entretenu. « Les deux grands poteaux qui, à quatre milles de Stoneham, marquaient l'entrée solennelle du chemin du LacSaint-Jean, n'avaient plus qu'un sens historique » (Tremblay, 1978: 146). Dix ans plus tard, le chemin de Saint-Urbain est abandonné et sera fermé à son tour. « Ce chemin dont la construction avait été entreprise en 1853 fut pratiquement abandonné en 1893 lorsque le chemin de fer reliant Québec au lac Saint-Jean arriva à Chicoutimi » (Saint-Aubin, 1987 : 11). Le gouvernement se serait-il trouvé des alliés pour accéder à ce territoire (Dussault, 1983)?

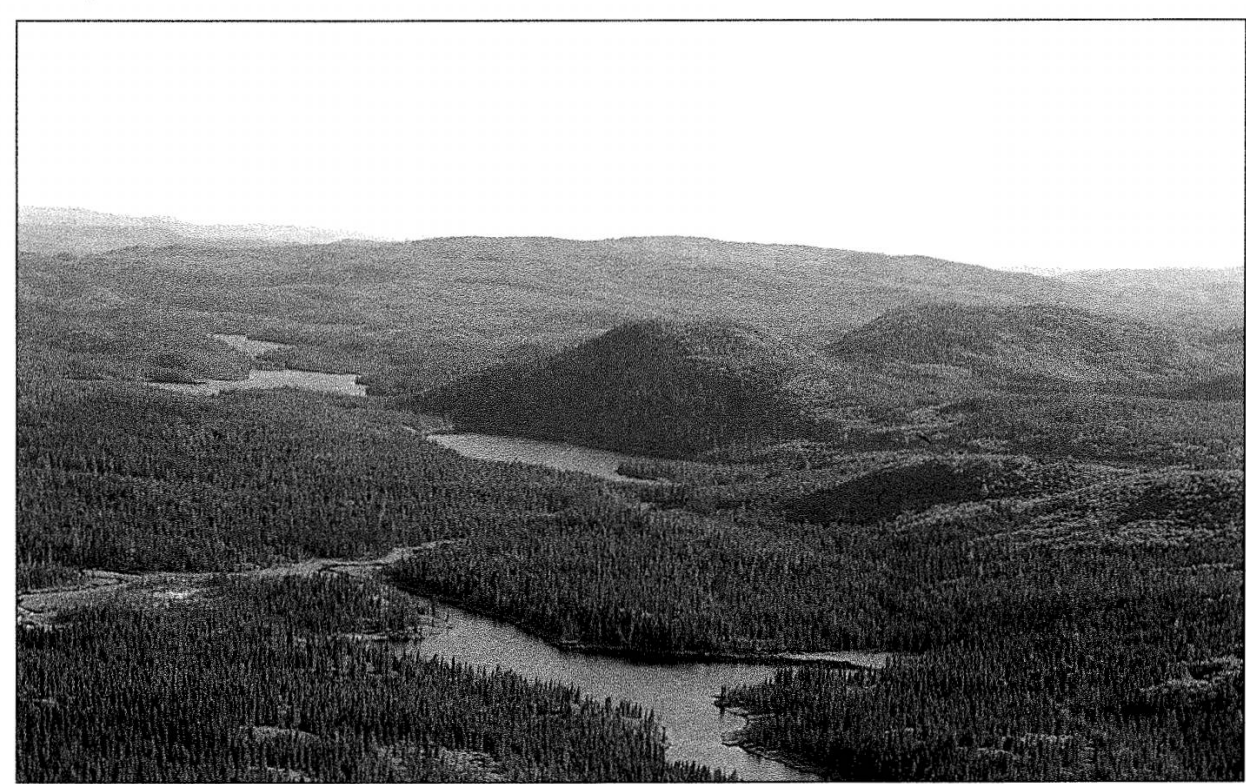

Vue aérienne du «Parc» des Laurentides, photo Pierre Lahoud.

Pendant près de quarante années, les seuls liens avec la région auront alors été par bateau via Tadoussac et le Saguenay et par train via Chambord et le lac Saint-Jean. On devra attendre la fin des années vingt pour que le gouvernement du Québec commence la construction d'une première route, entre Saint-Siméon et Grande-Baie (Demers, 1978 : 165). L'ouverture de cette artère a été rendu possible grâce à un programme « d'infrastructures » consacré au « retour à la terre » (Tremblay, 1978 : 145). En mai 1929, le Bulletin du ministère de la Voirie faisait ainsi la promotion de cette première « trajectoire » de pénétration :

Le district de Chicoutimi et du lac Saint-Jean sera cette année relié au reste de la province par un chemin amélioré actuellement en construction entre St-Siméon et Grande-Baie. Les travaux sont actuellement en cours et le chemin sera en assez bonne condition au cours de l'été (Québec, 1929 : 1).

Est-il possible d'avancer que, pendant le premier tiers du $\mathrm{XX}^{\mathrm{e}}$ siècle, on aurait « enclavé » volontairement ce territoire dans le but non avoué d'en restreindre l'accès ? La création du Parc national des Laurentides date de 1895, après l'affermage de nombreux clubs de chasse et de pêche dans le secteur. Il ne restait que ce territoire de libre. L'État aurait-il réservé ce territoire pour bloquer le passage, avant de le contrôler ? En 1922, l'auteur d'un guide touristique sur les Laurentides iden- tifie clairement la situation précaire due à la manœuvre d'enclavement de la zone :

Clubs land now from virtually a cordon around the park through which it is impossible for the poaching public to pass, since club guardians and park guards unite in an able vigilance-committee. [...] the park is rapidly becoming a sort of fountain of wild life which overflows its invisible boundaries to the benefits of the border-hunters (Longstreth, 1922 : 135).

Jean Désy a réalisé une étude sur les clubs privés de chasse et de pêche à l'époque de leur organisation le long de la ligne de chemin de fer. Il fait la remarque suivante sur leur localisation :

La carte [...] de Lemoine, [...] nous indique [que les clubs sont] tous axés le long du chemin de fer. Ce qui nous laisse supposer, parallèlement à la date des premiers écrits sur le sujet, que le chemin de fer de 1887 a réellement agi comme le facteur-clef du développement touristique entre Saint-Raymond et le Saguenay-LacSaint-Jean (1967 : 169).

Par ailleurs, l'auteur identifie la présence de clubs limitrophes au Parc des Laurentides : «Un club au territoire immense apparaît presque entièrement à l'intérieur du parc des Laurentides, le club Penn » (1967 : 173). Jules Dufour, quant à lui, émet des réserves sur l'objectivité des frontières du Parc (cité dans Saint-Aubin, 1987 : 22). Ces diverses préoccupations sur 1'enclavement du Parc étaient pourtant présentes dès 1923. À cette époque, les nouveaux administrateurs, le ministère de la Colonisation, des Mines et des Pêcheries, reconnaissaient d'emblée l'enclavement du territoire.

Ce parc créé dans le but d'en faire un endroit d'amusement et de récréation, a pratiquement été fermé au public ; excepté toutefois certaines réserves de chasse et de pêche qui ont été louées à des clubs ou à des particuliers le long des frontières Nord, Ouest et Sud afin de fermer l'accès et d'en rendre la protection plus efficace (Rapport général du ministère de la Colonisation, des Mines et des Pêcheries, 1923, cité dans CIFQ, 1974 : 19). 
Du même souffle, ces gestionnaires réaffirmaient la vocation d'origine du Parc.

\begin{abstract}
Nous avons commencé à organiser le Parc national des Laurentides, de façon à en faire une réserve de pêche et de chasse ainsi qu'un endroit d'amusement et de récréation pour le public, conformément à la pensée de ceux qui l'ont créé. Déjà, nous y possédons plusieurs maisons rustiques, dans des endroits les plus accessibles. Les demandes ont été si nombreuses pour la location de ces maisons qu'il nous faudra sans doute continuer à les multiplier (Ministère de la Colonisation, 1923 : 8).
\end{abstract}

En fin de compte, la réserve faunique des Laurentides sera accessible au public dans la mesure où sera renforcé le contrôle étatique :

- En 1924, l'État consolide sa juridiction sur le Parc des Laurentides et l'ouverture d'une route directe, « par monts et par vaux », est imminente. « D'ici peu de temps, aussi, une route classée sera ouverte entre la ville de Québec et le bas du Lac-Saint-Jean, permettant au tourisme américain de déferler vers ce pays nouveau pour lui, et dont la renommée 1'attire fortement. La maison de Maria Chapedelaine deviendra alors une sorte de Mecque pour les types innombrables genre Babbitt et Main Street, et cette seule raison pourrait justifier le soigneux entretien de la petite maison un peu négligée aujourd'hui » (Bilodeau, $1924: 83$ ).

- En 1929, on annonce officiellement que la route du «Parc » sera ouverte bientôt. «Il y a (aussi) une route en construction à travers le parc des Laurentides, entre Hébertville et Québec, mais les travaux ne sont pas encore assez avancés pour que la route puisse être livrée à la circulation cet été » (Québec, 1929 : $1)$.

- En 1930, le ministère de la Colonisation aménage de nombreux « camps » le long de la route. «Lorsque sera terminée la route qui traverse le parc national et qui reliera la région du Saguenay à la cité de Québec, nous aurons, le long de cette route, une vingtaine de petites hôtelleries bien aménagées, près de lacs poissonneux, pour recevoir les touristes et les amateurs de pêche » (Rapport général du ministère de la
Colonisation, 1930, cité dans CIFQ, $1974: 23$ ).

Bref, on vient d'assister à l'actualisation d'une trajectoire, à sa réalisation sous forme de route, enfin par son occupation économiquement viable. L'État vient d'établir son domaine, comme les autres acteurs, au XIX ${ }^{\mathrm{e}}$ siècle, l'avaient fait avec le LacSaint-Jean et le Saguenay.

\section{CONCLUSION}

Selon l'approche géographique structurale (Beaudet et Gagnon, 1999), l' analyse de la mise en scène du tourisme dans cette région a montré l'existence d'une différenciation régionale fondée sur un « contrôle du territoire », c'est-à-dire sur l'orientation de «trajectoires » éventuellement converties en flux touristiques. Dans cette perspective, il convient de tenir compte de l'attractivité des lieux et des rivalités entre acteurs qui déclenchent le déploiement de trajectoires engendrant des valorisations positionnelles différenciées. Les cas du Lac-Saint-Jean et du Saguenay sont des exemples éloquents de ce parcours.

Chargé de cours au Département de géographie de l'Université du Québec à Montréal, Serge Gagnon est étudiant au doctorat en géographie au CIEQ (Centre interuniversitaire d'études québécoises), Université Laval.

Un comité de lecture a lu et accepté ce texte

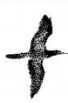

\section{NOTES}

1 Cet article représente des pistes de recherches dans le cadre d'un projet doctoral qui englobe une réflexion sur l'ensemble du Québec. Seuls les débuts de l'aventure au Saguenay-Lac-Saint-Jean sont ici approchés.

2 Pour cette première expérience, trois cent personnes auraient fait le voyage (Simard, 1987). Ce qui revient à dire que les touristes sont arrivés au Saguenay avant la vague massive d'immigration.

3 Le Saguenay ne peut être dissocié du domaine touristique du Bas-Saint-Laurent, communément appelé «Lower St. Lawrence ».

4 Le descriptif d' un guide touristique de cette compagnie est révélateur : From Niagara to the sea: descriptive of that delightful trip down the river St. Lawrence and up the worldfamed Saguenay. Richelieu and Ontario Navigation Co. (1896), Montréal.

\section{BIBLIOGRAPHIE}

Beaudet, G., et S. Gagnon (1999), « Esquisse d'une géographie structurale du tourisme et de la villégiature : l'exemple du Québec », sous la direction de N. Cazelais, R. Nadeau et G. Beaudet, L'espace touristique, Presses de l'Université du Québec, Sainte-Foy, p. 133-195.

Barbeau, M. (1938), «Saguenay », Canadian Geographical Journal, 16 (6), p. 285-291.

Beattie, B. (1995), Tadoussac: les sables d'été, souvenirs, histoires, légendes, Price Patterson ltée, Montréal.

Bilodeau, E. (1924), Autour du lac Saint-Jean : impressions de voyage, Éditions Casterman, Paris.

Buies, A. (1878), Petites chroniques pour 1877 , C. Darveau, Québec.

Buies, A. (1886), Sur le parcours du chemin de fer du Lac Saint-Jean, Imprimerie générale A. Côté et cie, Québec.

Buies, A. (1891), Au portique des Laurentides: une paroisse moderne, C. Darveau, Québec.

Corporation des ingénieurs forestiers de la province de Québec (CIFQ) (1974), Plein air et conservation au Québec, Comité des parcs en forêt.

Davies, B. (1930), Saguenay « Sâginawa»The River of Deep Waters, McClelland \& Stewart, Toronto.

Demers, A. (1978), « Le boulevard Talbot a trente ans », Saguenayensia, 20 (6), p. 165-171.

Désy, J. (1967), «Quelques aspects de la géographie des petites Laurentides de StRaymond », mémoire de maîtrise, Institut de géographie, Université Laval.

Dubé, P. (1986), Deux cents ans de villégiature dans Charlevoix, Les Presses de l'Université Laval, Québec.

Dussault, G. (1983), Le Curé Labelle : Messianisme, utopie et colonisation au Québec, 18501900, Hurtubise HMH, Montréal.

Gagnon, F. (1992), « Du cheval au rail : l'évolution des circuits touristiques québécois au $\mathrm{XIX}^{\mathrm{e}}$ siècle », Le pays laurentien au XIXe siècle, Cahier I, Université Laval, Université du Québec à Montréal, Université du Québec à TroisRivières, p. 101-133.

Gagnon, R. (1978), « Le chemin de fer Québec \& Lac-Saint-Jean », Saguenayensia, 20 (6), p. $152-164$.

Gagnon, S. (1996), « L'émergence du tourisme au $\mathrm{XIX}^{c}$ siècle, l'exemple de Charlevoix », Mémoire de maîtrise, Département de géographie, Université Laval. 
Gagnon, S. (1998), « De Lower St. Lawrence à Charlevoix », Téoros, 17 (1), p. 15-22.

Gaudreault, M. (1990), « Roberval », Continuité, 47-48, p. 47-51.

Knafou, R. et coll. (1997), « Une approche géographique du tourisme ", L'Espace géographique, 3, p. 193-204

Lebel, L. (1938), « Nos premiers vapeurs », Colon, 10 mars 1938.

Longstreth, M. T. (1922), The Laurentians: The Hills of the Habitants, McClelland \& Stewart Ltd., Toronto.

Lortie, A. (1993), « Hôtel Roberval : tout le monde descend!», Cap-aux-Diamants, 33, p. 54-57.

Macpherson Lemoine, J. (1872), L'album du touriste: archéologie, histoire, littérature, sport, Augustin Côté et Cie, Québec.

Pépin, P.-Y. (1969), Le royaume du Saguenay en 1968, Ottawa, Projet ARDA \# 15023, Direction générale du développement rural, Ministère de l'Expansion économique régionale, Ottawa.
Potvin, D. (1920), Le Tour du Saguenay : historique, légendaire et descriptif, Ministère de l'Agriculture, Québec.

Potvin, D. (13 édition-1946), The Saguenay Trip, Canada Steamship Lines, Montréal.

Québec, Ministère de l'Agriculture (1900), La Province de Québec, Département de l'Agriculture, Québec : 102-119.

Québec, Ministère de la Colonisation, des Mines et des Pêcheries (1923), Rapport général du ministère, Québec.

Québec, Ministère de la Voirie et des Mines (1928, 1929), Bulletin Officiel du Ministère de la Voirie, Bureau Provincial du Tourisme, 6 (811 ) et $7(1-4)$

QUÉBEC, Ministère de la Voirie (1934), Bulletin du Tourisme, Office Provincial du Tourisme, 1 (2).

Ritchot, G. (1999), Québec, forme d'établissement : étude de géographie régionale structurale, L'Harmattan, Paris.
Saint-Aubin, F. (1987), Histoire de la région des Grands Jardins - Tome 1, Ministère du Loisir, de la Chasse et de la Pêche, Direction régionale de Québec.

Simard, L. (1987), La petite histoire de Charlevoix, Club Lions de Clermont-La MalbaiePointe-au-Pic Inc.

Tittley, L. (1995), « Les croisières sur le SaintLaurent... un peu d'histoire » Téoros, 14 (2), p. 12-14.

Tremblay, V. (1978), « La route Québec-Saguenay », Saguenayensia, 20 (6), p. 142-147.

Trépanier, P. (1988), « Tadoussac », Continuité, 17 (1) p. 30-31.

Vien, R. (1954), Histoire de Roberval : cæur du Lac-Saint-Jean, Société historique du Saguenay, Chicoutimi.

Villeneuve, C. (1986), « La ouananiche en péril »Québec Science, p. 28-31.

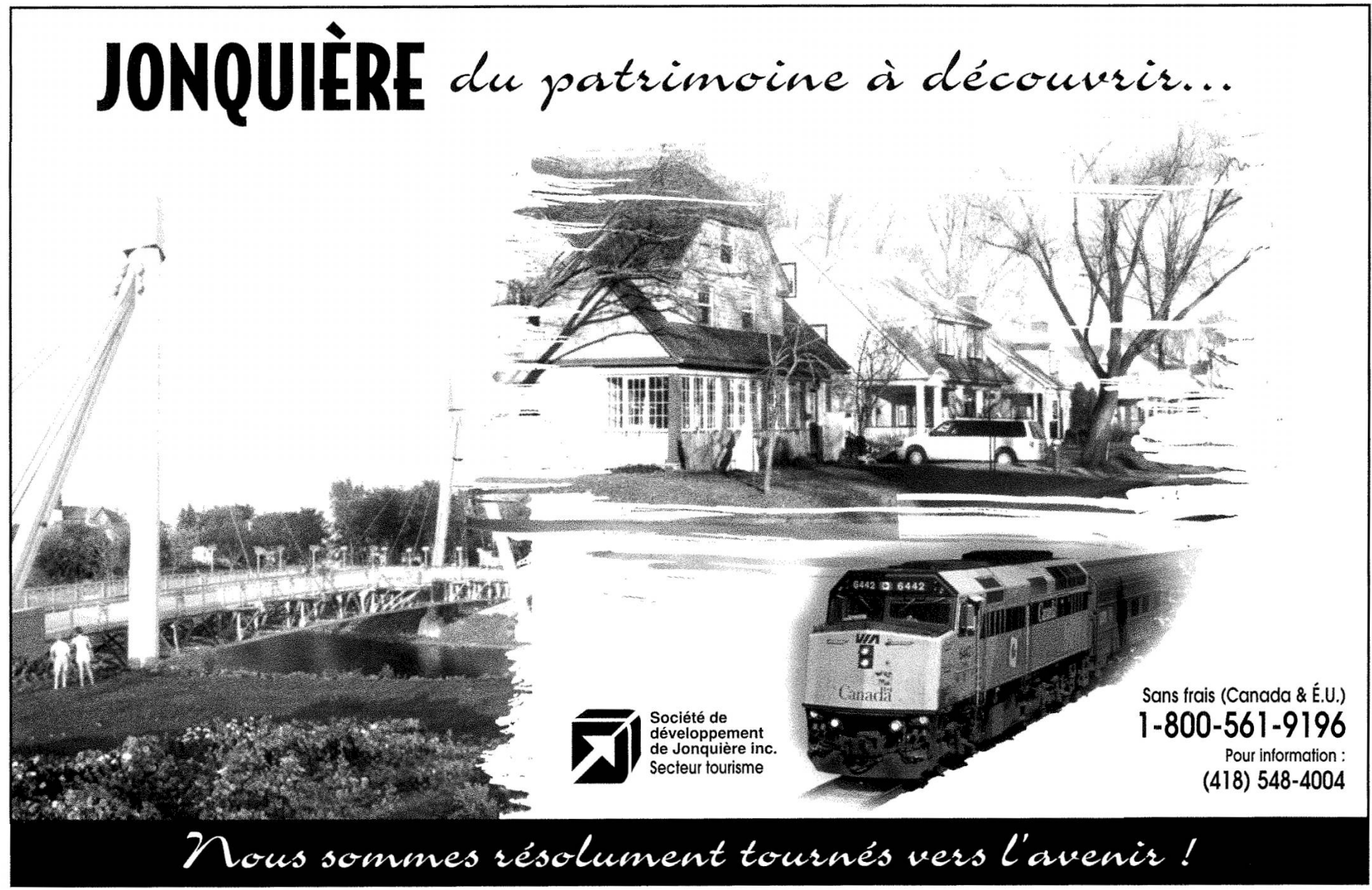

